\title{
Estrategias para fomentar la cultura tributaria desde la academia. Caso UTPL
}

\section{Strategies to promote the tax culture from the academy. UTPL case}

\author{
María Fernanda Yaguache Aguilar ${ }^{1 *}$, Mariuxi Pardo Cueva ${ }^{1}$ y Lupe Beatriz Espejo Jaramillo ${ }^{1}$ \\ ${ }^{1}$ Universidad Técnica Particular de Loja \\ *mfyaguache@utpl.edu.ec
}

DOI: https://doi.org/10.26871/killkana_social.v2i3.341

\begin{abstract}
Resumen
La finalidad de esta investigación es difundir las estrategias trabajadas desde la academia a través de los Núcleos de Apoyo Contable y Fiscal NAF, para fomentar la cultura tributaria responsable y voluntaria de las personas naturales no obligadas a llevar contabilidad en sectores vulnerables como: asociaciones de cafetaleros, adjudicatarios de los centros comerciales, artesanas de cerámica, asociaciones de la Mancomunidad Bosque Seco y de las Juntas Parroquiales de la Provincia de Loja. Las personas que forman parte de estos sectores carecen de conocimientos técnicos en el ámbito tributario y desarrollan sus actividades de manera empírica lo cual no les permite determinar de manera adecuada la rentabilidad de los productos que elaboran y por ende no cuentan con informes de los resultados económicos, factores que ocasionan un ambiente de desconfianza y desmotivación entre los miembros de las asociaciones. Estos sectores requieren de atención prioritaria no solo de los gobiernos seccionales sino también de la academia en virtud que desarrollan actividades económicas con productos propios de la localidad, por ello el interés de involucrar a estudiantes y docentes para fortalecer sus conocimientos, en el uso adecuado de los recursos mediante la aplicación de herramientas contables y administrativas básicas. La metodología utilizada es de tipo exploratorio-descriptivo; para levantar evidencias, se aplicó las técnicas de la encuesta y trabajo de campo, los datos se procesarón a través de métodos cuantitativos como el cálculo de frecuencias. De los resultados obtenidos se concluye que para fomentar la cultura tributaria se requiere de un trabajo conjunto entre la administración tributaria, la academia y la sociedad.
\end{abstract}

Palabras clave: Cultura tributaria, informalidad, Núcleos de Apoyo Contable y Fiscal, UTPL.

\begin{abstract}
The purpose of this research is to disseminate the strategies worked from the academy through the NAF Accounting and Fiscal Support Centers, to promote the responsible and voluntary tax culture of natural persons not obliged to keep accounts in vulnerable sectors such as: associations of coffee growers, adjudicators of shopping centers, ceramic artisans, associations of the Bosque Seco Commonwealth and of the Parochial Juntas of the Province of Loja. The people who are part of these sectors lack technical knowledge in the tax field and develop their activities in an empirical manner which does not allow them to adequately determine the profitability of the products they produce and, therefore, do not have reports on economic results. , factors that cause an atmosphere of distrust and demotivation among the members of the associations. These sectors require priority attention not only from the sectional governments but also from the academy by virtue of the fact that they develop economic activities with local products, therefore the interest of involving students and teachers to strengthen their knowledge, in the appropriate use of resources through the application of basic accounting and administrative tools. The methodology used is exploratory-descriptive; to gather evidence, the survey techniques and fieldwork were applied, the data was processed through quantitative methods such as frequency calculation. From the results obtained, it is concluded that to promote the tax culture, a joint work is required between the tax administration, academia and society.
\end{abstract}

Key words: Tax culture, informality, Accounting and Fiscal Support Centers, UTPL.

\section{Introducción}

La Fundación Interamericana para Iberoamérica de Administración y Políticas Públicas (2014) en la búsqueda de fomentar la inclusión de grupos vulnerables hacia el logro de mayores niveles de solidaridad diseña el Programa para la Cohesión Social en América Latina (EurosociAL) que trabaja en dos campos específicos: reducción de brechas y un mejor estado para garantizar derechos, redistri- 
buir y fiscalizar y de esta manera lograr una ciudadanía activa y participativa. Además, busca generar acuerdos de cooperación entre las administraciones tributarias y los centros de educación superior que fomenten la capacitación, seguimiento y asistencia permanente en información sobre normativa y procesos de índole tributaria, necesaria para la gestión de los centros Núcleos de Apoyo Contable y Fiscal (NAF), apoyando esta decisión, la administración tributaria de países latinoamericanos como Bolivia, Brasil, Costa Rica, Chile, Ecuador, Guatemala, México y Perú.

A este efecto, la Universidad Técnica Particular de Loja (UTPL), como centro de educación superior firmó un convenio de cooperación interinstitucional con el Servicio de Rentas Internas (SRI) en el año 2015, siendo su objetivo la creación de los NAF como centro gratuito de difusión del conocimiento contable - tributario, instruyendo a los beneficiarios sobre el sentido social de los impuestos y el cumplimiento de sus deberes formales como contribuyentes, con la participación de docentes y estudiantes de la Titulación de Contabilidad y Auditoría.

El acercamiento a los sectores vulnerables se efectúo a través de cuatro fases, lo cual permitió trabajar con diferentes segmentos de mercado, con necesidades similares. En estos sectores se pudo observó el bajo nivel de cultura tributaria, de organización y gestión contable y administrativa que mantienen las asociaciones, lo cual no les permite generar visibilidad de sus productos en el mercado local, así como acceder a créditos en las instituciones financieras y credibilidad en los organismos de control.

La metodología es de tipo exploratorio-descriptivo, para el levantamiento de la información se utiliza las técnicas: encuesta y el trabajo de campo, para el desarrollo de las actividades se establece cuatro fases: la planificación, preparación, implementación y evaluación, las mismas que fueron aplicadas en cada uno de los sectores intervenidos.

Se obtiene como resultados que en los sectores vulnerables existe una fuerte migración de la gente joven en busca de mejores oportunidades, quedando al frente de las asociaciones personas de edad avanzada y con una formación académica de nivel primario y secundario, factores que se convierten en desventaja para fomentar la cultura tributaria ya que existe desconocimiento de sus deberes formales como contribuyentes; sin embargo, una vez impartidos los talleres de capacitación, se motivan a los miembros de las asociaciones a llevar un control básico de sus registros contables.

\section{Marco teórico}

La cultura tributaria al ser un tema de interés a nivel mundial y mediante la determinación de políticas justas y equitativas cada gobierno blinda sus fronteras para evitar la fuga de capitales de su país hacia países de baja imposición fiscal. Por ello, se considera importante hacer referencia a los Objetivos de Desarrollo Sostenible (ODS) para el año 2017. El objetivo 16 permite disminuir las brechas de solicitudes de sobornos que involucra el no pago de impuesto, mientras que, el Objetivo 17 involucra por un lado, la implementación de aranceles para las importaciones y exportaciones entre países desarrollados y en desarrollo y por otra parte las remesas percibidas entre los países involucrados; situaciones que requieren de políticas coherentes, mayor compromiso a las alianzas y cooperación entre los actores.

Respecto a las prioridades de la Comisión Económica para América Latina CEPAL para apoyar a la implementación y seguimiento de la agenda 2030 para el Desarrollo Sostenible en los países de América Latina y el Caribe, en el ámbito fiscal se puede mencionar:

- El combate a los flujos ilícitos y la reducción de la evasión y elusión fiscal.

- El fortalecimiento de la acción e integración en las áreas productiva, comercial, tecnológica, fiscal, financiera e infraestructura.

- Potenciar la incorporación de los ODS en los sistemas de planificación nacional y territorial, incluidas las perspectivas de fiscalidad, presupuestos e inversión en el ámbito público.

- Meta del objetivo 10. Adoptar políticas fiscales, salariales y de protección social, y lograr progresivamente una mayor igualdad.

Para comprender el sentido de la cultura tributaria, se hace referencia a lo puntualizado por Fernandez (1998) quién señala que la cultura que no es una educación ni se da por adiestramiento; es decir, de una manera forzosa no se adquiere conocimientos de cultura, es un elemento que se comparte en sociedad en función a la educación recibida o el medio en el que se han desenvuelto la persona del cual dependerá su modo de pensar, criticar y distinguir lo que es bueno o malo.

Benalcazar (2015) considera que, la mayoría de los países latinoamericanos están buscando estrategias que les permita crear una cultura tributaria expresada como la conducta apropiada que se espera del contribuyente frente a la Administración Tributaria, desde la perspectiva ética, las obligaciones tributarias constituyen un deber moral fundado en la justicia legal. González (2006), se refiere al bajo índice de acción fiscalizadora que tienen algunos de estos países debido a que prefieren emplear sus limitados recursos al área de inspección que a exigir el cumplimiento de obligaciones tributarias de los grandes contribuyentes.

Artena y Auguste (Artena y Auguste, 2011) argumentan que, la informalidad tiene un efecto indirecto que puede afectar al crecimiento económico y la productividad de un país ya que el Estado reduce la posibilidad de financiar la provisión del gasto público de manera menos distorsiva posible.

Rivera y Sojo (2002) establecen que, la cultura tributaria no se puede entender como el conjunto de conocimientos que tienen los ciudadanos sobre las obligaciones y responsabilidades contributivas sino como la forma en que construyen una imagen de los impuestos a partir de una 
combinación de información y experiencias sobre la acción y el desempeño del Estado.

Méndez (2004) y Armas y Colmenares (2009) manifiestan que, la cultura tributaria es un conjunto de valores, conocimientos y actitudes compartidas por los miembros de una sociedad respecto a la tributación y la observancia de las leyes que la rigen, esto se traduce en una conducta manifestada en el cumplimiento permanente y voluntario de los deberes tributarios con base en la razón, la confianza, la afirmación de los valores de ética personal, los deberes $\mathrm{y}$ derechos que derivan para los sujetos activos y pasivos respeto a la ley, responsabilidad ciudadana y solidaridad social, tanto de los contribuyentes, como de los funcionarios de las diferentes administraciones tributarias, con la finalidad de crear una conciencia cívico-tributaria y así evitar la evasión fiscal.

Fernández (1998) propone a la administración fiscal que, para desarrollar una verdadera cultura tributaria, debe ir más allá que fijarse metas recaudatorias.

Cetrangolo y Gomez (2007) en la investigación realizada en 17 países, analizan que el grado de informalidad es creciente en los mercados especialmente laboral y de micro emprendimientos, lo que ha llevado a 14 países de la región a implementar un régimen especial de tributación para los pequeños contribuyentes quedándose exentos de estos sistemas Salvador, Panamá y Venezuela.

Para que el Estado pueda proveer de servicios básicos a la ciudadanía en general, debe diseñar estrategias que persuada a los grandes y pequeños contribuyentes al pago y cumplimiento de las obligaciones tributarias sin necesidad de acudir al poder coercitivo que por ley tiene derecho la administración tributaria (Castillo y Castillo, 2016).

Montero, (2013) considera que los incentivos fiscales son una buena estrategia de cultura tributaria para reducir la informalidad de los microempresarios y micro emprendimientos, estrategia que ha sido implementada tanto en Europa como Estados Unidos.

La universidad ecuatoriana es humanista, cultural y científica, bajo este sentido debe reconocer, fortalecer y defender cada grupo social y la comunidad Quintanilla2012(Quintanilla, 2012).

Para hacer referencia al fortalecimiento de la institucionalidad, la Ley Orgánica de Educación Superior en el Art. 124 responsabiliza a las instituciones de educación superior proporcionar a los egresados de cualquier carrera, el conocimiento efectivo de los deberes y derechos de los ciudadanos y de la realidad socioeconómica, cultural y ecológica del país.

En Ecuador, la educación en cultura tributaria es prioritario en sus tres niveles: primario, medio y secundario porque la solidaridad y equidad es un deber en la formación de los seres humanos con un nuevo pensamiento de ciudadanía fiscal para logra el desarrollo del país, al respecto Carrasco, (2010) conceptualiza como la "conciencia de las personas, grupos e instituciones, acerca de su responsabilidad y compromiso con la construcción de una sociedad cohesionada, democrática y justa, a través del ejercicio de sus derechos y deberes fiscales" . y Manzur (2012) y Grant, Mejía y García (2015) consideran que, la base para lograr concientización fiscal en los contribuyentes es desde la formación inicial de manera que los futuros profesionales sean conscientes que el aporte solidario del pago de impuestos generará un mejor ambiente territorial basado en el compromiso, la legalidad y solidaridad en el cumplimiento de obligaciones tributarias.

Todo ciudadano está en la obligación moral de aportar solidariamente sus obligaciones tributarias, sean estas desde el plano empresarial, comercial, profesional, autónomo, relación de dependencia, entre otros; es por eso transcendente la formación de planes de capacitación por sectores económicos para el cumplimiento tributario; es decir, se resalta la importancia de diseñar para cada uno de estos sectores un modelo curricular basado en la Ética - Cohesión Social - Pacto Social.

Según Estrada (2014) los elementos de la cultura tributaria son: El conocimiento. (Conocer y entender los principales aspectos de la tributación que forman parte de la cultura tributaria); La percepción (que viene a ser, la sensación, ideas u opiniones en materia fiscal, suelen relacionarse tanto con la recaudación de los tributos como con su utilización) y el Comportamiento tributario (Actitudes y prácticas de conducta, corresponden al pensar y al sentir de sus miembros, cuya reiteración las convierte en hábitos, costumbres o tradiciones). En el caso de la cultura tributaria se expresa en la práctica y puede medirse por el grado de cumplimiento voluntario, no se limita al "saber" sino trasciende al "hacer"; es decir, no se trata de un tema conceptual sino conductual. Esto se relaciona con lo afirmado por Valero y Briceño (2010) cuando hace referencia a los valores de los contribuyentes como: la responsabilidad, la cooperación, el compromiso y la solidaridad, que unificados crean la relación sociedad - gobierno.

La legislación del Ecuador establece los deberes formales, obligaciones y derechos de los contribuyentes, considerados en el Código Tributario, emitido por el Congreso Nacional (2005) y modificado por la Asamblea Constituyente (2016); así como, en la Circular No. NACDGECCGC17-00000004, emitida por el SRI (2017). Respecto de las obligaciones de los contribuyentes, se hace referencia a los principales: Presentar declaraciones de impuestos y la obligación de llevar contabilidad, existen dos tipos de contribuyentes que quedan exentos, las personas naturales no obligadas a llevar contabilidad; las personas naturales que se acogen al Régimen Impositivo Simplificado RISE. Mientras que al hacer referencia a los derechos de los contribuyentes se puede mencionar: de trato y confidencialidad, de asistencia y colaboración, económicos y procedimentales.

Asimismo, la Constitución de la República del Ecuador, en su sección quinta, el Régimen Tributario, en el Art. 300, menciona que el régimen tributario se regirá por los principios de generalidad, progresividad, eficiencia, 
simplicidad administrativa, irretroactividad, equidad, transparencia y suficiencia recaudatoria.

La obligación del Estado es propender la mejor distribución de la riqueza, que está en concordancia con el objetivo 8 del Plan Nacional del Buen Vivir (PNBV), política 8.2 que señala: "Consolidar el papel del estado como dinamizador y regulador del mercado"; resaltando el lineamiento $8.2 \mathrm{~b}$ referente $\mathrm{a}$ :

Optimizar el gasto tributario y aplicar incentivos tributarios para la producción, el empleo, la reinversión de capitales y la sostenibilidad biofísica, en función del cumplimiento de criterios de desempeño y la incorporación de trabajo local y el componente nacional en el proceso productivo, manteniendo criterios de progresividad (Secretaría Nacional de Planificación , 2007, p.20).

Una de las principales reformas tributarias a la Ley de Régimen Impositivo Simplificado del Ecuador dirigido a los microempresarios, los exime de efectuar declaraciones para el cumplimiento de sus obligaciones tributarias, por el pago de una cuota acorde al nivel de ingresos por actividad económica que desarrollen, este sistema ha sido implementado con la finalidad de disminuir la informalidad de los contribuyentes. Asamblea Nacional Constituyente (R.O. 242-3S-XII-2007).

El Estado para evitar prácticas monopólicas, impulsar un comercio justo, mejorar la productividad y la producción sostenible, ha diseñado incentivos fiscales, que se han estructurado en generales, sectoriales y para el desarrollo regional equitativo y para zonas deprimidas, los cuales se ajustan a la realidad económica de cada sector. Asamblea Nacional Constituyente (R.O 351 de 29-dic-2010).

El Centro de Estudios Fiscales del Ecuador del Servicio de Rentas Internas, cuenta con el área de cultura tributaria, encargada de:

- Diseñar programas de educación en Fiscalidad en los tres niveles educativos: Cultura Tributaria para la Educación Básica, Educación y Capacitación Tributaria dirigido a estudiantes de bachillerato y el pasaporte tributario dirigido especialmente a profesionales y estudiantes de nivel superior.

- Crear el portal Cívico Tributario, en el que se difunde el día Nacional de la Cultura Tributaria, que se celebra el 27 de abril.

- Fomentar la investigaciones a través de la Revista Fiscalidad,

- La marca de ciudadanía fiscal, con incentivos como la lotería tributaria.

- Firma de convenios con centros de educación superior para impulsar la creación de los NAF como centros gratuitos de difusión de la cultura tributaria.

La Administración Tributaria de cada país dentro de un proceso de formador de formadores de Ciudadanía Fiscal, están en la obligación de extender el conocimiento a través de gestores de la información en cada uno de sus pares estudiantes - formadores que permitan identificar cuáles son los objetivos de la sociedad, de la universidad y de los administradores de impuestos y hacia dónde van (Vargas, 2014).

Según datos del Instituto Nacional de Estadísticas y Censos INEC del Ecuador refleja que para el mes de marzo de 2016, el $32.6 \%$ de las personas trabajan en establecimientos que no se encuentran registrados ante la administración tributaria bajo el Registro Único de Contribuyentes RUC para el desarrollo de sus actividades comerciales.

Para reducir este porcentaje de informalidad, la Administración Tributaria inicia campañas publicitarias para fomentar en las MyPyMES la inscripción o cambio del régimen general al RISE, generando un crecimiento ascendente a partir de la creación de esta ley como se observa en figura 1.

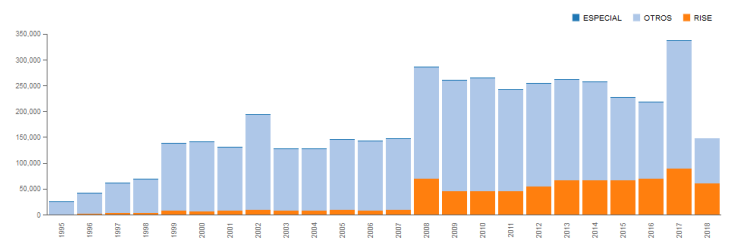

Figura 1. Inscripción de los contribuyentes en el RUC y RISE.

Fuente: https://declaraciones.sri.gob.ec/saiku-ui/

La Administración Tributaria desde el año 2015 impulsa la implementación de los Núcleos de Apoyo Contable y Fiscal (NAF) con las instituciones de educación superior, bajo convenio de cooperación interinstitucional.

La atención que brindan los estudiantes es gratuita y debe ser de calidad que motive a los contribuyentes al cumplimiento voluntario y oportuno de las obligaciones tributarias. La Administración Tributaria para ejercer el control y seguimiento de los proyectos ha diseñado estándares de presentación de los informes con la finalidad de generar evidencia del accionar de las partes involucradas.

El SRI mantiene convenios de cooperación con las siguientes universidades del Ecuador:

Tabla 1 Convenio de cooperación SRI - Universidades del Ecuador.

\begin{tabular}{|c|l|}
\hline Localidad & \multicolumn{1}{|c|}{ Universidad } \\
\hline Quito & Pontificia Universidad Católica del Ecuador \\
\hline Quito & Universidad Central del Ecuador \\
\hline Quito & Universidad de las Fuerzas Armadas del Ecuador \\
\hline Quito & Universidad Tecnológica Equinoccial \\
\hline Sto. Dt. & Universidad Autónoma Regional de los Andes \\
\hline Ambato & Universidad Autónoma Regional de los Andes \\
\hline Cuenca & Universidad Politécnica Salesiana \\
\hline Cuenca & Universidad Católica del Cuenca \\
\hline Guayaquil & Universidad Politécnica Salesiana \\
\hline Guayaquil & Instituto Tecnológico Superior Almirante Illingworth \\
\hline Loja & Universidad Técnica Particular de Loja \\
\hline
\end{tabular}

Fuente: Centro de Estudios Fiscales - NAF 
Cada una de las universidades antes mencionadas diseñan sus propios proyectos de cooperación para la comunidad, todas bajo un mismo fin, fomentar la cultura tributaria en el Ecuador.

\section{Metodología}

La Universidad Técnica Particular de Loja UTPL con el afán de impulsar el convenio firmado con la Administración Tributaria por un periodo de 5 años, involucra a los estudiantes de la Titulación de Contabilidad y Auditoría para dar cumplimiento a las obligaciones estipuladas en el convenio. A partir del año 2015 la UTPL desarrolla un proyecto de vinculación con la sociedad, acogiéndose a lo establecido en el Art. 125 de la LOES relacionado con la formación de programas y cursos de vinculación con la sociedad.

Los involucrados por parte de la UTPL, son los estudiantes y profesores de la Titulación de Contabilidad y Auditoría y desde la Administración Tributaria los funcionarios, quienes en conjunto deben organizar diferentes temáticas de actualización tributaria y de interés a los sectores vulnerables que sean seleccionados para desarrollar el proyecto de vinculación correspondiente.

La metodología utilizada es de tipo exploratorio- descriptivo, para obtener evidencias, se aplica técnicas de levantamientos de información, la encuesta y trabajo de campo, los datos se procesan a través de métodos cuantitativos como el cálculo de frecuencias.

El trabajo de campo fue desarrollado en siete periodos académicos ordinarios y extraordinarios desde el año 2015 hasta el año 2017. Se aplicó las 4 fases establecidas en la figura 2.

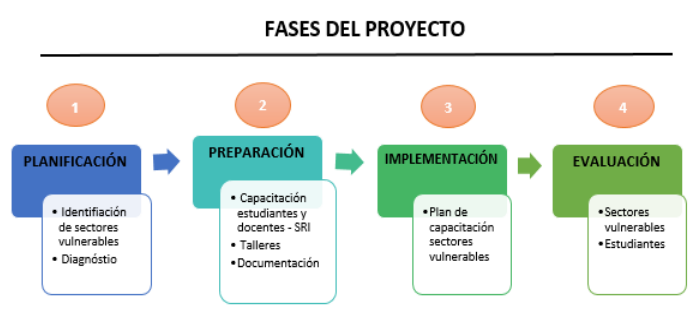

Figura 2. Fases del proyecto de vinculación

Fuente: Proyecto de vinculación

Los procedimientos y/o actividades establecidas en cada una de las fases, se presentan a continuación.

\subsection{Fase 1: Planificación}

Se identificaron los sectores vulnerables que permitieron a los estudiantes el desarrollo de las actividades planificadas.

Los sectores vulnerables identificados cumplieron con el requerimiento inicial de la Administración Tributaria de ser personas naturales no obligadas a llevar contabilidad.
En la tabla 2 se especifica los sectores vulnerables y el periodo académico de ejecución del proyecto de vinculación.

Tabla 2 Identificación de sectores vulnerables

\begin{tabular}{|l|c|}
\hline \multicolumn{1}{|c|}{ Sectores Identificado } & $\begin{array}{c}\text { Período Académico de } \\
\text { Ejecución }\end{array}$ \\
\hline $\begin{array}{l}\text { Personas naturales no obligadas a llevar con- } \\
\text { tabilidad }\end{array}$ & Enero - abril 2015 \\
\hline $\begin{array}{l}\text { Cafetaleros de Vilcabamba, Quinara y Palan- } \\
\text { da artesanos de Cera y Cachipamba }\end{array}$ & Abril - agosto 2015 \\
\hline $\begin{array}{l}\text { Adjudicatarios de los mercados: Centro co- } \\
\text { mercial Loja, Reina del Cisne y Gran Colom- } \\
\text { bia }\end{array}$ & $\begin{array}{c}\text { Octubre 2015 - febrero } \\
2016\end{array}$ \\
\hline $\begin{array}{l}\text { Personas naturales no obligadas a llevar con- } \\
\text { tabilidad, atendidas en la oficina de asesoría } \\
\text { contable. }\end{array}$ & Febrero - marzo 2016 \\
\hline $\begin{array}{l}\text { Socios de las asociaciones de la Mancomuni- } \\
\text { dad Bosque Seco. }\end{array}$ & Abril - agosto 2016 \\
\hline $\begin{array}{l}\text { Asociaciones de las juntas parroquiales de } \\
\text { Loja }\end{array}$ & Octubre 2016 - febrero \\
\cline { 2 - 2 } & Abril-Agosto 2017 2017 \\
\hline
\end{tabular}

Fuente: Informes de cumplimiento del proyecto.

Respecto a los sectores involucrados se observa que, al ser personas de avanzada edad, el nivel de rusticidad con el que desarrollan sus actividades y su nivel bajo de formación académica, presentan resistencia a involucrarse en el cumplimiento de sus obligaciones tributarias, Sin embargo, por temor a ser sancionados por parte de la Administración Tributaria existe un gran interés por participar de las asesorías y conocer cuáles son sus deberes y obligaciones tributarias.

\subsection{Fase 2: Preparación}

De acuerdo a las necesidades de los contribuyentes del sector identificado, se solicita a los funcionarios delegados del Servicios de Rentas Internas la preparación de material y capacitación en temas tributarios a los estudiantes y docentes de la Titulación de Contabilidad y Auditoría de la UTPL.

Para el cumplimiento de esta fase se seleccionó grupos de 15 a 20 estudiantes matriculados en los componentes académicos de gestión productiva 2, 3.1 y 3.2. Asimismo, se asignó dos docentes de la Titulación para que brinden apoyo en cada una de las fases establecidas para el proyecto. La capacitación tributaria se desarrolló en 20 horas presenciales, debiendo ser el curso aprobado mediante una evaluación de conocimientos.

Por otro lado, con el apoyo de docentes y estudiantes de la Titulación, se elaboró material didáctico a ser utilizado en los talleres dirigidos a los sectores vulnerables.

\subsection{Fase 3: Implementación}

En esta fase se ejecutó el plan de capacitación para cada uno de los sectores identificados en las siguientes temáticas: deberes formales de los contribuyente; RUC y RISE; comprobantes de venta; proceso de matriculación vehicular; traspaso de dominio y exoneraciones; Impuesto 
al Valor Agregado; facturación electrónica; gastos personales; impuesto a la renta; herencias, legados y donaciones; retenciones de impuesto a la renta y de IVA; infracciones; devolución de IVA para tercera edad y discapacitados; manejo de la herramienta virtual del SRI y Régimen Simplificado para asociaciones bajo el control de la Superintendencia de Economía Popular y Solidaria (SEPS).

Los estudiantes desarrollaron esta fase bajo criterios de capacitación, asesoría y acompañamiento a los sectores involucrados y con orientaciones de tutores internos (docentes) y tutores externos (funcionarios SRI e involucrados de las Juntas Parroquiales y Municipio de Loja).

En la tabla 3, se presenta el número de estudiantes involucrados y usuarios atendidos por cada sector vulnerable identificado.

Tabla 3 Estudiantes capacitados y usuarios atendidos

\begin{tabular}{lcc}
\hline \multicolumn{1}{c}{ Sectores identificados } & $\begin{array}{c}\mathbf{N}^{\circ} \mathbf{d e} \\
\text { estudiantes }\end{array}$ & $\begin{array}{c}\mathbf{N}^{\circ} \mathbf{d e} \\
\text { usuarios } \\
\text { atendidos }\end{array}$ \\
\hline $\begin{array}{l}\text { Personas naturales no obligadas a llevar } \\
\text { contabilidad }\end{array}$ & 15 & 90 \\
$\begin{array}{l}\text { Cafetaleros de Vilcabamba, Quinara y } \\
\text { Palanda Artesanos de Cera y Cachipam- }\end{array}$ & 7 & 102 \\
ba & & \\
$\begin{array}{l}\text { Adjudicatarios de los mercados: Centro } \\
\text { Comercial Loja Reina de El Cisne y } \\
\text { Gran Colombia }\end{array}$ & 19 & 351 \\
$\begin{array}{l}\text { Personas naturales no obligadas a llevar } \\
\text { contabilidad, oficina de asesoría contable }\end{array}$ & 4 & 750 \\
$\begin{array}{l}\text { Socios de las asociaciones de la Manco- } \\
\text { munidad Bosque Seco. }\end{array}$ & 6 & 33 \\
$\begin{array}{l}\text { Asociaciones de las Juntas Parroquiales } \\
\text { de Loja }\end{array}$ & 24 & 22 \\
\hline Total & $\mathbf{7 5}$ & $\mathbf{1 3 4 8}$ \\
\hline
\end{tabular}

Fuente: informes de cumplimiento del proyecto.

\subsection{Fase 4: Evaluación}

Para cuantificar los resultados obtenidos en el proceso de capacitación y asesoría a los diferentes sectores involucrados, se toma como caso de estudio las Asociaciones de la Mancomunidad Bosque Seco.

Para determinar el nivel de conocimientos en la implementación del proyecto, se aplicó una evaluación relacionada con temas tributarios, en dos momentos: antes de la capacitación y posterior a la capacitación.

\section{Resultados}

Respecto al género y edad se establece que en la capacitación tributaria dictada por los estudiantes de la Titulación de Contabilidad y Auditoría de la UTPL participaron 16 hombres y 17 mujeres de los cantones de Celica y Macará (Ver tabla 4)
Tabla 4 Género de los participantes

\begin{tabular}{cccc}
\hline Cantón & Masculino & Femenino & $\%$ \\
\hline Celica & 6 & 7 & 48 \\
Macará & 10 & 10 & 52 \\
\hline Total & 16 & 17 & $100 \%$
\end{tabular}

Fuente: Encuesta

La edad de los participantes de las asociaciones, oscila entre los 31 y 50 años con un $37 \%$ y un $13 \%$ de los socios tiene edades entre los rangos de 20 a 30 años y más de 50 años. (Ver tabla 5)

Tabla 5 Edad de los participantes

\begin{tabular}{lcccc}
\hline \multicolumn{1}{c}{ Opciones } & Celica & Macará & Total & $\%$ \\
\hline Menos de 20 años & 0 & 0 & 0 & 0 \\
De 20 a 30 años & & 4 & 4 & 13 \\
De 31 a 40 años & 6 & 7 & 13 & 38 \\
De 41 a 50 años & 5 & 7 & 12 & 36 \\
Más de 50 años & 1 & 3 & 4 & 13 \\
\hline Total & 12 & 21 & 33 & 100
\end{tabular}

Fuente: Encuesta

El $55 \%$ de los socios de la Mancomunidad del Bosque Seco, poseen una formación de nivel secundaria y un $42 \%$ de nivel primario (Ver tabla 6). Como se puede observar es el resultado de la edad promedio que tienen las personas encuestadas, por ende responden a una época en la que los estudios no eran fundamentales y por la actividad económica a la que se dedicaban tampoco les permitía acceder a un nivel universitario de formación.

Tabla 6 Edad de los participantes

\begin{tabular}{lcccc}
\hline \multicolumn{1}{c}{ Opciones } & Celica & Macará & Total & $\%$ \\
\hline Primaria & 3 & 11 & 14 & 42 \\
Secundaria & 10 & 8 & 18 & 55 \\
Universitaria & 0 & 1 & 1 & 3 \\
\hline Total & 13 & 20 & 33 & 100
\end{tabular}

Fuente: Encuesta

El $70 \%$ de los socios de la Mancomunidad del Bosque Seco, se dedica a actividades relacionadas con la agricultura, sobresaliendo la producción de café y maíz; el $18 \%$ se dedica a actividades comerciales como la venta de productos artesanales y agroecológicos y el $12 \%$ restante se dedica a la producción apícola y de lácteos (Ver tabla 7).

Tabla 7 Actividad a la que se dedica

\begin{tabular}{lcccc}
\hline \multicolumn{1}{c}{ Opciones } & Celica & Macará & Total & $\%$ \\
\hline Agricultura & 10 & 13 & 23 & 70 \\
Comercio & 2 & 4 & 6 & 18 \\
Ganadería & 0 & 0 & 0 & 0 \\
Otras & 1 & 3 & 4 & 12 \\
\hline Total & 13 & 20 & 33 & 100
\end{tabular}

Fuente: Encuesta 
Para determinar los resultados de la capacitación, se unificó las temáticas tributarias impartida a los estudiantes de la Titulación de Contabilidad y Auditoría de la UTPL y por parte del SRI y sea utiliza como herramienta para la capacitación a los participantes en cuatro temas generales que se indican en la tabla 8 , resultados que demuestran el impacto positivo y de interés que generar una cultura tributaria responsable y voluntaria en las personas naturales no obligadas a llevar contabilidad, de las asociaciones de la Mancomunidad de Bosque Seco.

Tabla 8 Capacitación en temática tributaria

\begin{tabular}{lcccc}
\hline \multirow{2}{*}{ Temática } & \multicolumn{2}{c}{ 1ra. encuesta en \% } & \multicolumn{2}{c}{ 2da. encuesta en \% } \\
& $\mathrm{Si}$ & $\mathrm{No}$ & $\mathrm{Si}$ & $\mathrm{No}$ \\
\hline Información del RUC & 30.75 & 69.25 & 95.60 & 4.40 \\
Comprobantes de venta & 40.00 & 60.00 & 90.60 & 9.40 \\
Impuestos & 36.25 & 63.75 & 76.40 & 23.60 \\
Servicios en línea & 12.75 & 87.25 & 82.67 & 17.33
\end{tabular}

Fuente: Encuesta

Respecto a la capacitación en temas tributarios relacionados con información del RUC, utilización e importancia de los comprobantes de venta, cumplimiento en la declaración de los impuestos y uso de la plataforma virtual del SRI para servicios en línea, se obtiene un incremento entre la fase inicial y final de la capacitación pasando del $30.75 \%$ al $95.60 \%$; del $40.00 \%$ al $90.60 \%$; del $36.25 \%$ al $76.40 \%$ y del $12.75 \%$ al $82.67 \%$, respectivamente.

La difusión de resultados obtenidos, se efectúo a través de los siguientes medios:

- Canal de comunicación interna de la UTPL

- Redes sociales de la UTPL

- Pantallas internas del Servicio de Rentas Internas.

- Entrega de informes de actividades desarrolladas a cada uno de los sectores intervenidos.

\section{Conclusiones y recomendaciones}

El presente trabajo se desarrolló con la finalidad de difundir las estrategias aplicadas y los logros alcanzados por la UTPL al fomentar los NAF desde a academia, lo cual ha generado enriquecimiento de saberes para estudiantes, docentes, funcionarios del SRI y especialmente de los sectores que han sido intervenidos para crear competencias en el cumplimiento de sus obligaciones tributarias.

Se considera necesario continuar con los Núcleos de Apoyo Contable y Fiscal NAF, brindando asesoría tributaria de manera gratuita que permita a la ciudadanía en general conocer la importancia de los impuestos para el desarrollo del país.

Para la Titulación de Contabilidad y Auditoría es un gran aporte en el proceso de formación de sus estudiantes, ya que adquieren las competencias necesarias para involucrarse como futuros Contadores Públicos en la sociedad y generar información financiera que cumpla con la normativa tributaria vigente.
Los sectores vulnerables presentan una gran debilidad, como es la ausencia de jóvenes; lo cual disminuye el interés de acceder a capacitaciones frecuentes impartidas por la Administración Tributaria en beneficio de sus emprendimientos.

El gobierno debería impulsar planes de beca de formación profesional a los jóvenes de cada uno de estos sectores, con la finalidad que, al término de su formación presenten un plan de trabajo o una propuesta de mejora para impulsar la producción y comercialización de los productos con estándares de calidad.

Los proyectos de vinculación que desarrollan las Instituciones de Educación Superior deberían ser elaborados y ejecutados de forma conjunta entre los Organismo de Control y Gobiernos Seccionales con la finalidad que los requerimientos de información a los sectores vulnerables sean solicitados de manera organizada y que aporte de forma efectiva a las necesidades de los beneficiarios.

Los Gobiernos Seccionales deberían diseñar planes de seguimiento, evaluación y control del crecimiento económico que están obteniendo las asociaciones con la maquinaria donada por las entidades públicas, factores que motivarían a los asociados a mantenerse constantemente capacitados y de esta manera el involucramiento de la academia generaría un mayor valor agregado.

\section{Referencias Bibliográficas}

Armas, M. E., y de Eizaga, M. C. (2009). Educación para el desarrollo de la cultura tributaria. REDHECS: Revista electrínica de Humanidades, Educación y Comunicación Social, 4(6), 141-160. Descargado de https://dialnet.unirioja.es/ servlet/articulo? codigo=2937210

Artena, D., y Auguste, S. (2011). Informalidad, productividad y crecimiento. propuesta metodológica basada en censos industriales. Banco Interamericano de Desarrollo. (Notas técnicas, IDB-TN-342, 1-103.)

Benalcazar, J. (2015). Elementos de la cultura tributaria en el ecuador. cultura contributiva en américa latina. Delegación Benito Juarez. C.P. 03100, México, D.F..

Carrasco V., C. M. (2010). La ciudadanía fiscal. (Fiscalidad, SRI, 56, 11-67)

Castillo, S., y Castillo, P. (2016). Un acercamiento al estudio de la cultura tributaria desde la perspectiva socioeducativa. Dominio de las Ciencias, 2(3), 149162.

Cetrángolo, O., y Gómez, J. (2007). La tributación directa en américa latina y los desafíos a la imposición sobre la renta. CEPAL-Macroeconomía del desarrollo Serie.

Estrada, S. (2014). Cultura tributaria: 50 preguntas y respuestas. Superintendencia de Administración Tributaria.

Fernández, R. (1998). La cultura tributaria frente a la cultura empresarial: propuesta para alcanzar una 
conciliación fiscal. Revista de Ciencias Sociales, 4(1).

Grant, M., Mejía, F., y García, E. (2015). La concientización fiscal de los contribuyentes, como base para formar la cultura tributaria en méxico. Ciencia Administrativa, 2, 144-150.

Meza, C., y Manzur, K. (2012). Estrategias de comunicación y búsqueda de alianzas para los programas de educación fiscal de américa latina. Instituto de Estudios Fiscales.

Méndez, M. (2004). Cultura tributaria, deberes y derechos vs. constitución de 1999. Revista Espacio Abierto, 13(1), 123-137.

Montero, M. (2013). Aspectos fiscales de las donaciones a entidades sin fines de lucro en estados unidos. Revista de Economía Pública, Social y Cooperativa, 79, 193-218.

Quintanilla, J. (2012). La universidad en la cultura tributaria. Retos, 2(3), 105-114.

Rivera, R., y Sojo, C. (2002). Cultura tributaria. el sistema tributario costarricense. (Inf. Téc.). Contribuciones al Debate Nacional. San José, Contraloría General de la República.

Secretaría Nacional de Planificación . (2007). Plan nacio- nal del buen vivir 2007- 2014 (Inf. Téc.). Secretaría Nacional de Planificación. Descargado de www . sri - gob.ec

Valero, M., y Briceño, F. (2010). Ética y cultura tributaria en el contribuyente. Revista Daena, 5(1). (International Journal of Good Conscience)

Vargas, J. (2014). Ciudadanía fiscal en la formación por competencias. Revista Fiscalidad, 8.

Recibido: 30 de junio de 2018

Aceptado: 3 de septiembre de 2018 